\title{
Defining 'disease'-classification must be distinguished from evaluation
}

\author{
P D Toon Merton College, Oxford
}

\section{Author's abstract}

The use of the term 'disease' in medicine is discussed, with particular reference to the issues raised by Kennedy (I) and the definition proposed by Campbell, Scadding and Roberts (2). Certain difficulties arising from this definition are considered, and a revised set of definitions is suggested, based on a distinction between diseasedness, contrasted both with health and with other sorts of problems, and nosological categories used to distinguish conditions calling for different treatments. The difference is stressed between those aspects of medical decision-making which call for judgment on scientific grounds and those of the sort referred to by Kennedy, which involve ethical and political judgments.

The use of the terms 'disease' and 'diseases' has come under increasing scrutiny in recent years, in response to the need to define terms precisely and overtly in the field of computer-aided diagnosis, and to criticism of the 'medical model' of disease in psychiatry by some clinical psychologists, and by so-called anti-psychiatrists. This debate was highlighted when Kennedy ( $I$ ) chose to start 'unmasking medicine' by a consideration of the terms 'illness' and 'disease'.

He considered the two terms separately, in the former stressing the evaluative nature of the notion of ill-health, and in the latter pointing to some weaknesses he saw in the 'mechanical' analogies of disease. He did not however attempt to examine closely the relationship between the sociallyconditioned value judgments involved in decisions about illness, and the technical distinctions made between different diseases, nor how the disease theory he criticises can be improved to overcome some of the weaknesses of which he accuses it.

An important contribution to this discussion was the definition of disease proposed by Scadding (3), and revised by Campbell, Scadding and Roberts (2). In the latter paper, and the correspondence which followed it, it became yet more apparent that clear ideas on these terms basic to medicine, are important in a wide range of clinical issues. In this article an attempt will be made to advance the debate by considering various aspects of the definition adopted in that paper, and to incorporate the conclusions drawn into alternative definitions.

\section{Disease and diseases}

Campbell et al (2) distinguish between what they call 'essentialist' and 'nominalist' concepts of disease. Though their use of this terminology has been criticised (4) the point of their distinction, that it makes more sense to think of a disease as a useful term in medical discourse for a phenomenon or group of phenomena, rather than as an agent causing illness, is an important one. The disadvantages associated with the essentialist view have been discussed by Kennedy (I), Campbell et al (2) and elsewhere (5), and do not require restating. However the 'nominalist' definition Campbell et al suggest raises several problems. They propose that:

... in medical discourse, the name of a disease refers to the sum of the abnormal phenomena displayed by a group of living organisms in association with a specified common characteristic or set of characteristics by which they differ from the norm of their species in such a way as to place them at a biological disadvantage.

This definition fails to distinguish two quite different ways in which the word 'disease' is used in both medical and colloquial discourse. First, there is the dichotomy between disease and non-disease, in which the term implies that the state of the organism is in some way undesirable, and requires if possible to be changed; and that a medical intervention is the appropriate way to attempt this. Secondly, there is the quite different need to distinguish one disease from another, for the purpose of treatment and prognosis. To ask 'is X ill ?' is quite different from asking 'what illness does $\mathrm{X}$ have ?' though clearly these questions are related. The distinction may be clarified by considering two groups of problems caused by the lack of a clear concept of disease which have been given as examples previously (5).

The first involved arguments about the defining characteristics of chronic bronchitis, asthma and emphysema, the other concerned the dispute about whether or not certain conditions such as homosexuality, alcoholism and psychopathy are rightly considered diseases.

Though superficially similar, the two problems on closer consideration in fact differ greatly. In the first case what is at issue is the definition of a particular disease; how do we decide what asthma is, in order to say that patient $\mathrm{X}$ has asthma rather than 
bronchitis or emphysema. There is no doubt that $\mathrm{X}$ is ill; the question is what illness does he have. It is a problem of categorisation.

The cases in the second group, however, concern disease in general contrasted with non-disease, rather than a distinction between disease $A$ and disease $\mathrm{B}$. There is frequently no dispute as to the definition of the category; everyone may agree that $\mathrm{X}$ is an alcoholic, a psychopath or a homosexual; what is at issue is whether such a condition is properly called a disease. Campbell et al (2) seem to assume that by defining what is a disease they will automatically define disease, presumably on the assumption that disease is merely the sum of all possible diseases. However there are conditions which whilst clearly defined in the same terms as diseases, are not at all disadvantageous (for example the Dubin-Johnson syndrome, an abnormality of liver enzymes which has no symptoms, and is usually detected on routine blood tests) and there are disadvantageous conditions (poverty was an example which was used by Campbell et al (2)) which it is not useful to consider as diseases. Non-disease includes both health and non-medical problems. Thus in framing our definitions it is necessary to consider what is meant by a disease, what we mean by disease in general, and on what grounds the latter is distinguished from health and from non-medical problems.

\section{Biological disadvantage and abnormality}

A second problem with their definitions is the use of the term 'biological advantage'. The problems arising from this term have been raised before (6), (7). While at first sight it seems an adequate criterion, attempts to apply it rigorously (8) lead to conclusions which seem ridiculous, such as that voluntary celibacy is a disease state, or that elective sterilisation is an iatrogenic illness. The reason why the notion of biological disadvantage leads to such surprising conclusions is that doctors do not concern themselves with maximising the evolutionary advantages of the human race as a whole, but with aiding individuals. Similarly patients go to their doctors not because they believe themselves to be evolutionarily disadvantaged, but because there is some state of affairs which they wish altered or explained, and they feel the doctor is the appropriate person to do that. It is not, as Kennedy (I) suggests, always the doctor who judges a state to be an illness; the vast majority of medical consultations are initiated not by the doctor but by the patient. A large part of medicine is concerned with aiding those who, in evolutionary terms, have outlived their usefulness, being of neither economic nor reproductive value to society. Similarly many disease states, though of great inconvenience to the individual concerned, have no influence, or only a trivial one, on success defined in evolutionary terms; for example minor, non-life-threatening illnesses such as colds, sinusitis and the 'chronic nuisance' sort of diseases such as eczema, psoriasis, and warts. The formulation of individual disadvantage woudid seem to approach more closely to what doctors doo

What, however in this context do we mean disadvantage ? An implied advantage of the concept of biological disadvantage seems to be that provides a clear criterion for determining what doctors should do, thus freeing medicine from the need to become involved in confusing and difficuft areas of moral philosophy. However as is clear fro $\vec{m}$ the examples above, it does this at the cost of distorting the concept of medicine away from whe doctors actually do. Furthermore, the ethical judgment is not avoided, it is merely concealed within the notion of biological disadvantage. Thits Kendell's (8) operational definition of biologica disadvantage in terms of shortened life and decrease fertility is merely a covert way of decreeing that prolonged life and fecundity are desirable goals, an that medicine should strive to achieve these. Th tendency to find moral values creeping in under the cloak of a scientific definition is a central problem 윽 the definition of disease, and it merely leads to confusion. The solution which will be adopted here is not to attempt to offer a system of moral values for medicine, either descriptively or prescriptively, but to use the word 'disadvantage' where such mora judgments are required, as a sort of 'master car\$ which can be replaced by whatever concept of the $\mathrm{Bad}$ is held by the reader. The consideration of what constitutes a disadvantage, $i e$ what states on $\vec{E}$ should seek to modify and which to achieve, is question which would require separate, and length consideration.

As Kennedy (I) rightly points out, decisions of this issue are conditioned by prevalent values and social norms, and doctors have no special expertis? on these matters. Generally medical judgments reflect social consensus, and the example he gives, of the American Psychiatric Association's decision to remove homosexuality from its list of diseases, is $\mathrm{a}$ good instance of this. The decision clearly reflects the influence of the considerable change in general attitudes to homosexuality in American society iff the sixities and early seventies.

The use of the terms 'abnormal' and 'norm' in the definition is also unsatisfactory, on the same grounds. They must either be defined in a way that makes the definition exclude cases which clearlo come within commonly held concepts of disease, o\$ which carry concealed within them a value judgment of what is good and desirable. Definitions in terms of statistical abnormality come into the first class since there often arise situations, such as parasitie infections in some tropical countries where the majority are affected, but which would usually be considered diseases. Furthermore, the supposition that the desirability of altering a state depends eve 
partly on its statistical abnormality carries within it a moral prejudice in favour of conformity which is, to say the least, questionable. Such ideas as a 'biological norm' presuppose that what is biological is good and desirable, again leading either to the acceptance of a rosy view of a 'state of nature', or to uncritical satisfaction with some contemporary ideal.

\section{Three questions, not one}

The attraction of such terms as 'abnormal' and 'biological disadvantage' is that they appear to offer firm and scientific ways of distinguishing disease from health, and deciding when a medical intervention is the appropriate one, and when a problem is someone else's business. These are certainly vital questions, because it is certainly necessary for a doctor to know what it is his duty to try to treat. The problem arises from a confusion between logical problems of categories and their relationships, and ethical problems. It becomes clearer if instead of asking a single question 'what is a disease ?' which is the one Campbell et al (2) have attempted to answer, one asks three separate questions:

I) How do we decide whether an individual's state is good or bad?

2) If the state is in any way undesirable, how do we decide that it is undesirable in a way that indicates that medical intervention is required ?

3) What is the nature of the categories which doctors use to divide up phenomena and which they generally call diseases?

In the light of the above discussion, it would seem preferable to answer these questions in a way which avoided the use of the concepts of abnormality and biological disadvantage.

The first is clearly recognisable as a traditional question of moral philosophy, and at first sight seems to have little to do with everyday clinical practice. In the vast majority of cases there is little dispute; except in extraordinary circumstances broken legs and inflamed appendices are regarded as desirable by almost no-one. It is however in the difficult cases that definitions like Scadding's are of use, and it is in difficult cases that they are most severely tested $(I, 3)$. The attempt to solve an ethical decision by recasting the problem in terms of one of scientific definition can only cloud issues. It is perhaps significant that it is in areas where this consensus breaks down, such as psychiatry, that the definition of disease often is seen as a problem. As indicated above, the nature of the Good and of Evil in a medical context, though clearly of vital importance, is such a large question that rather than consider it inadequately, I shall not discuss it further.

The second question, though clearly not independent of the first, can to some extent be con- sidered separately. What, then, defines the proper area of medical concern? This has varied from period to period; for example at one time medicine and surgery were considered to be separate professions in a way that they are not now. Patients who are now referred to psychiatrists have in other ages been sent to priests for exorcism, and certain sorts of psychiatric patient are now treated by psychologists rather than psychiatrists. What is considered a medical problem is not immutable, but neither is it as arbitrary as Kennedy (I) seems to suggest. It will depend on the medical options offered and the alternatives available. One pragmatic way of answering the question is in terms of how doctors' special training and skills, and medical knowledge as it exists today, is oriented. What are doctors good at ? Contemporary medicine is based on the analysis of problems in terms of a group of disciplines of which the two foundations are anatomy and physiology. The other medical disciplines such as immunology, biochemistry, pharmacology, pathology etc are all closely related to these two and interrelated one with another. They share a common mechanistic model, in which function, both normal and abnormal, at a variety of levels of organisation is interpreted in terms of structure, from gross anatomy to electron microscopy. It is in the analysis of problems in terms of this model that doctors are trained. It therefore seems reasonable, and not excessively circular, to define diseases as problems (according to whatever moral system one prefers) which are suitably analysed in terms of this model, which I will call the 'anatomico-physiological model'. Clearly there will be disputes in grey areas, as for example when a psychologist may favour a behavioural analysis and treatment, whilst a psychiatrist may interpret the same problem in biochemical terms and offer drug therapy. The difference, however, is over means not ends, and is of a different type from disagreements about whether a state is satisfactory or requires altering.

This is not to say that doctors should only concern themselves with analysis in these terms, an approach which Kennedy (I) criticises; good medicine will not lose sight of the person in the analysis of the problem, and will recognise the need for help with other aspects from relatives, friends, nurses, social workers etc., and not forget the patient's own contribution. Nevertheless in this role the doctor is acting as a sympathetic human being, albeit one who is professionally sympathetic, rather than exercising his professional skill, and the concepts of disease are a tool for the use of that skill. Considering the first two questions separately makes it much simpler to consider the nature of the categories used by doctors to organise data (symptoms, signs, investigation results, etc) into patterns which recur and so enable past experience to be used in future judgments. 


\section{Nosological category}

To avoid confusion, the term nosological category is suggested for these concepts, since they are, literally, categories used in the study of disease. As Campbell et al (2) suggest, these categories can be compound, defining the state of the organism in terms of two or more sets of categories, such as a morbid anatomical one - pneumonia - and a microbiological one - varicella infection - to give the diagnosis of varicella pneumonia. Sets of categories may be orthogonally related, as in this example, so that definition in terms of one set is independent of the other, a full definition depending on both, as a point on a map is defined by latitude and longitude. They can also be hierarchical, as for example when one defines a pathophysiological state, such as left ventricular failure, and then proceeds to seek for an underlying cause, such as hypertension, myocardial infarction, cardiomyopathy, etc. Though traditionally diseases have been thought of as discrete, box-like categories, there is a growing tendency to think more in terms of continuous dimensions, particularly in diseases with multifactorial aetiology. Medicine is leaving behind the idea, criticised by Kennedy ( $I$ ) of 'specific diseases caused by specific agents', if indeed it was ever held.

Thus perhaps one can think of obstructive airways disease in terms of three continuous dimensions of emphysema, chronic bronchitis and asthma, with each patient occupying a particular point in the space defined by these dimensions, as on a three dimensional graph, according to how severely he or she is affected by each process. Similarly the 'classical' pictures of polymyalgia rheumatica and temporal arteritis are now seen by some physicians, not as defining boxes into which we must try to fit recalcitrant clinical pictures, but as defining the ends of a dimension on which clinical cases may fall. In choosing useful nosological categories, it is important to bear in mind their purpose, to aid decisions as regards treatment and prognosis. If two conditions do not differ in treatment or prognosis then there is little value in distinguishing them for 'a difference that makes no difference is no difference'. Of course differences which do not appear to be important from these points of view may repay exploration by increasing our understanding, and new knowledge may make it important to divide a category formerly considered homogeneous (as has for example happened to infective hepatitis in recent years). Though naturally most of the categories and dimensions will be diseases in the first sense, since the ultimate purpose in studying them is to seek to treat them, this is not a necessity of the categorisation. There are anatomico-physiological states, such as the Dubin-Johnson syndrome mentioned above, which while statistically abnormal and defined in the same categories as diseases, confer no real disadvantages and therefore in no way merit treatment. This is not a new idea; surgeons and anatomists have long characterised some of the wide variations of the human organism as anomalieg rather than diseases. In some cases, hypertension being a good example, a dimension will stretch from full health to life-threatening disease, and the point at which to draw a line distinguishing health from disease is to some extent arbitary and is based on a analysis of the advantages and disadvantages to the organism of medical intervention, again partly an ethical matter.

Clearly the distinctions between the three questions made here are artificial and they are complexly interrelated, and of course in real life are usually considered together. It is, however, imports ant to contrast intellectual judgments (whic $\mathbb{R}$ disease pattern does this case most clearly resemble which drug is most likely to have a certain effect on a patient ?) from ethical or value judgments (would this person be better off if not homosexual; is the cost of this treatment worth it for the benefits it bestows?). Though the discussion has focussed of the diagnostic stage of a medical consultation, the same distinctions are relevant to treatment decisions as indicated in the examples above.

\section{Conclusions}

In the light of the above discussion, definitions two levels are offered, one of being diseased and one of a nosological category or dimension. Therefore?

I) A nosological category is defined in terms of the anatomico-physiological model by one or mor $\vec{E}$ features displayed by organisms within that category. If the variables are continuous rather than discrete, it may often be more valuable to think in terms of a dimension, rather than a set of separate categories.

2) An organism is said to be diseased when it fallg into a nosological category, or in a position on nosological dimension, which places it at a 'dis advantage' in comparison with an organism which differs only in the features of that nosologicat category or dimension.

If the difference between organisms within and without the nosological category is not such as to place it at a disadvantage, the expression 'variant' of 'anomaly' may be used.

It is important to note that these are functionab definitions, and as such are only intended to be of use in the limited context of potential or actual medicaf consultation. They do not attempt to answer widef? philosophical questions which may arise outside thiso context, such as whether or not when a lay persorp takes an aspirin for a hangover he is treating disease. Unlike previous formulations, no attemp $\mathbb{D}$ is made to avoid the value judgments in the categori sation of an organism as diseased, and in the need for medical intervention. It is hoped that bye distinguishing the cognitive aspects of categorisation 
from the moral considerations of diseasedness, and offering an empirical approach to the question of the proper scope of medicine, confusion and the justification of moral values on apparently scientific grounds will be avoided.

\section{References}

(I) Kennedy I. The Reith Lectures: Unmasking medicine. The Listener 1980 Nov 6, 13, 20, 27, Dec 4, II.

(2) Campbell E M, Scadding J G, Roberts R S. The concept of disease. British medical journal 1979; 2: 757-762.
(3) Scadding J G. Diagnosis, the clinician and the computer. Lancet 1967 ; ii: 877-882.

(4) Kraupl-Taylor F. Concepts of disease. Psychological medicine 1980; 103: 419-424.

(5) Editorial. The concept of disease. British medical journal 1979; 2: 75I-752.

(6) Toon P D. Psychiatry and the concept of disaese. British journal of psychiatry 1976; 128: 99.

(7) Toon P D. Correspondence: the concept of disease. British medical journal 1979; 2: 1001.

(8) Kendell R E. The concept of disease and its implications for psychiatry. British journal of psychiatry 1975; 127: 305-313. 